\title{
Enhanced superprism effect in symmetry reduced photonic crystals
}

Cite as: Appl. Phys. Lett. 113, 131103 (2018); https://doi.org/10.1063/1.5032197

Submitted: 03 April 2018 . Accepted: 01 September 2018 . Published Online: 26 September 2018

M. Gumus, I. H. Giden (D), O. Akcaalan, M. Turduev, and H. Kurt
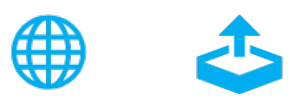

Export Citation

\section{ARTICLES YOU MAY BE INTERESTED IN}

Performance of quantum-dot-based tunnel-injection lasers: A theoretical analysis

Applied Physics Letters 113, 131101 (2018); https://doi.org/10.1063/1.5045860

Topologically induced transparency in a two-phase metamaterial

Applied Physics Letters 113, 131106 (2018); https://doi.org/10.1063/1.5042577

$17000 \% / W$ second-harmonic conversion efficiency in single-crystalline aluminum nitride microresonators

Applied Physics Letters 113, 131102 (2018); https://doi.org/10.1063/1.5042506

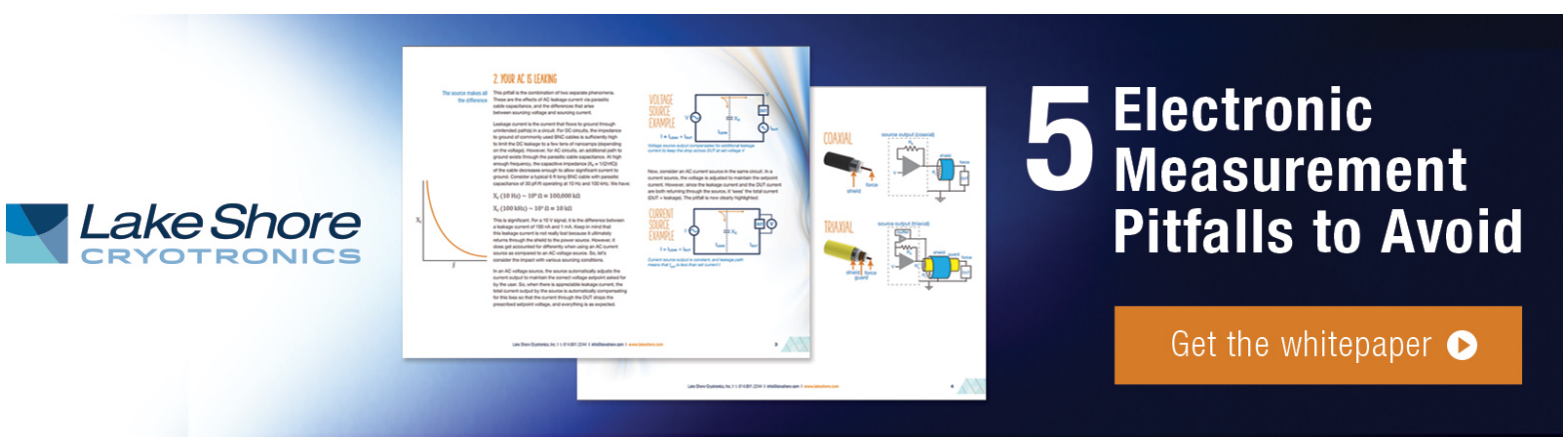




\title{
Enhanced superprism effect in symmetry reduced photonic crystals
}

\author{
M. Gumus, ${ }^{1, a)}$ I. H. Giden, ${ }^{1}$ O. Akcaalan, ${ }^{2}$ M. Turduev, ${ }^{3}$ and H. Kurt ${ }^{1}$ \\ ${ }^{1}$ Nanophotonics Research Laboratory, Department of Electrical and Electronics Engineering, \\ TOBB University of Economics and Technology, Ankara 06560, Turkey \\ ${ }^{2}$ Department of Physics, Bilkent University, Ankara 06800, Turkey \\ ${ }^{3}$ Department of Electrical and Electronics Engineering, TED University, Ankara 06420, Turkey
}

(Received 3 April 2018; accepted 1 September 2018; published online 26 September 2018)

\begin{abstract}
We propose compact $\mathbf{S}$-vector superprism providing broadband wavelength sensitivity within $a$ / $\lambda=0.610-0.635$, where " $a$ " is the lattice constant, $\lambda$ is the incident wavelength, and $\boldsymbol{S}$ denotes the Poynting vector. The reported configuration overcomes strong beam divergence and complex beam generation due to the self-collimation ability of the low symmetric primitive photonic crystal ( $\mathrm{PhC})$ cells. Analytical calculations of equi-frequency contours, photonic band structures, and group velocity dispersions are performed by solving Maxwell's equations and using the plane wave expansion method. Besides, finite-difference time-domain analyses are also conducted. The designed superprism induces large refracted angle variation for different frequencies when the incident angle is fixed: $4 \%$ change of incident frequencies results in approximately $40^{\circ}$ deflected angle difference with a maximum $68.9^{\circ}$ deflection angle inside the $\mathrm{PhC}$. Meanwhile, for a fixed incident wavelength, a large output variation occurs if the incident angle is altered. Microwave experimental results are found to be in good agreement with the numerical analyses. Published by AIP Publishing. https://doi.org/10.1063/1.5032197
\end{abstract}

Superprisms can be described as a wavelength sensitive optical beam deflection effect including two unique optical properties: super-dispersion and angular magnification. ${ }^{1}$ Like a conventional prism, superprism structures are able to spatially separate different wavelengths of the incident beam, the so-called "super-dispersion." In the case of a single wavelength of incident light, the superprism steers the propagating beam with a wider angle when the incident angle is slightly changed. This condition explains the angular magnification property. Such a strong incident angle-dependent beam steering effect could be attributed to the strong modifications of the group velocity $\left(\boldsymbol{v}_{\boldsymbol{g}}\right)$ existing due to the index modulation inside photonic crystal $(\mathrm{PhC})$ structures. ${ }^{2}$

Group velocity vector $v_{g}=d \omega / d k$ expresses the direction of the Poynting vector, i.e., the direction of energy flow of light. A superprism structure deflecting the direction of propagating light inside the $\mathrm{PhCs}$ is called the $S$-vector superprism. On the other hand, a wave vector $(|\boldsymbol{k}|=2 \pi / \lambda)$ indicating the phase front propagation of light and a superprism structure that utilizes the phase velocity $\left(\nu_{p}=c / k\right)$ dispersion is called $\boldsymbol{k}$-vector superprism, which is able to deflect the beam outside the PhCs according to Snell's law. ${ }^{1,3}$ The angle of outgoing light from the $\mathrm{PhCs}$ /air interface depends on the boundary conditions and $\boldsymbol{k}$-vector. The angles of incoming and outgoing light should be the same due to the $\boldsymbol{k}$-vector conservation ${ }^{4}$ and thus, inclined output ends should be introduced to the $\boldsymbol{k}$-vector superprism to enable wavelength resolution. ${ }^{3,5}$ On the other hand, $S$-vector superprisms are relatively bulky structures compared to their $\boldsymbol{k}$-vector counterparts to separate completely the wavelength components of the propagating beam inside the PhCs. Moreover, beam broadening is a big obstacle for $\boldsymbol{S}$-vector superprisms, which need super-lenses to suppress the unwanted beam diffraction. ${ }^{4}$

\footnotetext{
a)Author to whom correspondence should be addressed: mgumus@etu.edu.tr
}

While an optimal superprism satisfies the requirements such as wavelength sensitivity and wide-angle magnification, beam divergence and irregular beam generation may exist as an inconvenience in $S$-vector superprisms. ${ }^{6}$ In order to avoid undesired beam divergence through the beam propagation, a self-collimation based superprism structure could be designed. Also, the resolution of the designed superprism can be enhanced, which is a crucial criterion for superprism efficiency and it depends on the wavelength and angle sensitivity to enhance the superprism effect. ${ }^{7}$

At the normalized frequency $\tilde{\omega}=\omega / 2 \pi c=a / \lambda$, the optical performance of superprisms can be investigated by calculating the following three parameters: (1) beam broadening factor, $p=\partial \theta_{c} / \partial \theta_{i},(2)$ dispersion factor, $q=\partial \theta_{c} / \partial \tilde{\omega}$, and (3) the resolution parameter, $r=q / p .^{7}$ In these relations, $\theta_{c}$ represents the propagation angle of the refractive beam inside the PhCs and $\theta_{i}$ is the incident angle. ${ }^{7,8}$ In a recent study, ${ }^{9}$ another parameter named angular-group dispersion-bandwidth-product is defined as a criterion of the superprism effect for spectroscopic applications. In Ref. 10, authors attempted to increase the wavelength sensitivity by using hetero $\mathrm{PhCs}$ with oblique boundaries for efficient wavelength demultiplexing applications. ${ }^{10}$

A low symmetry feature that constructs the main part of the work is considered, we can refer to Ref. 11, which has similar features to the concept. Even though the study ${ }^{11}$ numerically examines anomalous diffractive characteristic (tilted self-collimation effect) due to introduced structural asymmetry to the $\mathrm{PhC}$ structure, in this work differently from that report we present the super-dispersive phenomenon that shows superprism and self-collimation effects simultaneously which is the main goal of the work. In other words, the proposed structure is wavelength sensitive, incident angle sensitive, and has diffraction limited light propagation characteristic. Also, experimental verification of the superprism effect is provided in the microwave regime in the presented study. 
Apart from the previous studies, in this paper we present a beam deflecting $S$-vector superprism made of low-symmetric PhCs. The proposed superprism structure is compact compared to its $S$-vector superprism counterparts so that it could be implemented for dense photonic integrated circuits.

Wavelength sensitive beam deflection in the proposed structure is conceptually explained in Fig. 1(a): White light is incident to the proposed low-symmetric PhCs with an incident angle $\theta_{\text {in }}$ and its wavelength components are deflected in different angles enabling spatial wavelength separation. Top-view of the proposed configuration is also illustrated in Fig. 1(a) with important geometrical parameters. The refractive index of $\mathrm{PhCs}$ is 3.1, and the unit cell includes $\mathrm{PhC}$ rods with different radii.

The effect of symmetry reduction in PhCs could be analytically explored to calculate the corresponding photonic band structures and equi-frequency contour (EFC) calculations as well as to better understand light manipulation inside the PhCs. Maxwell's equations can be solved by the plane wave expansion method to obtain solutions of photonic band structures. ${ }^{12-14}$ Master's equations for $\mathbf{E}$-polarization and $\mathbf{H}$ polarizations can be described as eigen-equations shown below

$$
\sum_{\mathrm{G}^{\prime}}|\mathbf{k}+\mathbf{G}|\left|\mathbf{k}+\mathbf{G}^{\prime}\right| \varepsilon^{-1}\left(\mathbf{G}-\mathbf{G}^{\prime}\right) H_{\perp, \mathbf{k}}\left(\mathbf{G}^{\prime}\right)=\frac{\omega^{2}}{c_{0}^{2}} H_{\perp, \mathbf{k}}(\mathbf{G})
$$

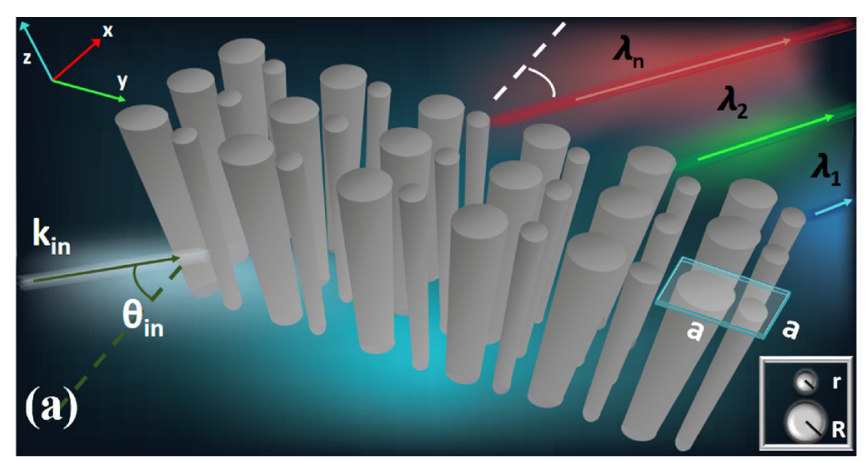

(b)
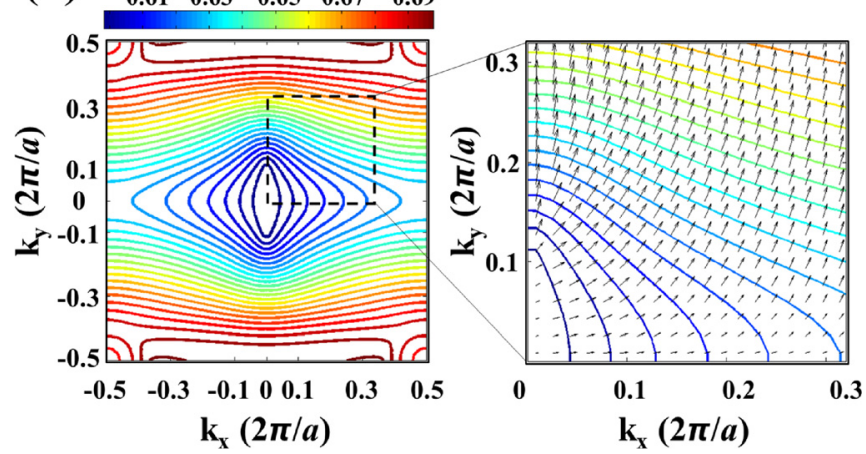

FIG. 1. (a) 3D representation of the low symmetric square-lattice PhC structure as a self-collimation superprism. The incident light direction is indicated with wave-vector $\boldsymbol{k}_{\text {in }}$ and $\theta_{\text {in }}$ demonstrates the angle of incidence. The propagating beam deflects inside the PhCs with respect to wavelengths; $\lambda_{1}$, $\lambda_{2}$, and $\lambda_{\mathrm{n}}$ and the corresponding unit cell is given as an inset; $\{R, r\}$ $=\{0.20 a, 0.10 a\}$ are the radii of smaller and larger dielectric PhC rods, respectively. (b) Calculated fourth band EFCs of TM polarization for the $C_{1}$-symmetric $\mathrm{PhC}$ superprism structure. The zoomed section indicates the EFCs at which a strong superprism effect exists and the arrows show the direction of Poynting vectors.
$\sum_{\mathrm{G}^{\prime}}(\mathbf{k}+\mathbf{G})\left(\mathbf{k}+\mathbf{G}^{\prime}\right) \varepsilon^{-1}\left(\mathbf{G}-\mathbf{G}^{\prime}\right) H_{\|, \mathbf{k}}\left(\mathbf{G}^{\prime}\right)=\frac{\omega^{2}}{c_{0}^{2}} H_{\|, \mathbf{k}}(\mathbf{G})$.

Detailed analytical derivations for given eigen-equations to obtain photonic band structures are provided in the supplementary material. Photonic band structures for both E-polarization and $\mathbf{H}$-polarization are calculated on MATLAB ${ }^{15}$ by using the plane wave expansion method on Maxwell's equations. Here, E-polarization is a transverse-electric (TE) mode where the electric field components are along the $x y$-plane $\left(\mathrm{E}_{x}, \mathrm{E}_{y}\right)$ and the magnetic field $\mathrm{H}_{\mathrm{z}}$ is perpendicular to the $x y$-plane [corresponding axis representation are given as an inset in Fig. 1(a)]. On the other hand, considered H-polarization is a transversemagnetic (TM) mode where the non-zero electric and magnetic field components are defined as $\mathrm{E}_{z}, \mathrm{H}_{x}$, and $\mathrm{H}_{y}$, respectively. It is important to note that throughout the study only the TM polarization mode is considered.

Symmetry reduction is introduced in the rotational symmetry ${ }^{11}$ of the PhC unit cell in this study. A regular (cylindrical) unit cell is the trivial case with isotropic behavior. We used $C_{1}$ symmetry in our study to show the intended superprism effect. Even though $C_{2}$ symmetric PhCs possess the superprism at studied frequencies, the strongest superprism effect appears in the $C_{l}$ case, which is the main reason for selecting $C_{I}$ symmetry for efficient superprism applications. Introducing more modifications in the $\mathrm{PhC}$ unit cell by adding smaller $\mathrm{PhC}$ rods into it, the superprism effect disappears despite existing constant beam deflection regardless of the frequency variations (see the supplementary material). In the presented work, $\mathrm{C}_{1}$-symmetric square lattice $\mathrm{PhCs}$ are considered in this study due to their existing strong superprism effect because adjustable dielectric distribution of the elementary cell due to $C_{1}$ symmetry increases the degree of freedom which is a tool for providing different light manipulation techniques. The proposed $\mathrm{PhC}$ design allows the dielectric density to vary according to the distances and angles to each other of the different radii dielectric rods, permits a reproducible change in the dielectric functions, and guides the light accordingly. Also the comparison between the rod-type and hole-type of the suggested structure is investigated (see the supplementary material).

The designed structure is composed of cylindrical alumina rods having a permittivity value of $\varepsilon=9.61$ and radii of $\{R, r\}=\{0.20 a, 0.10 a\}$, which corresponds to a filling ratio of $f=0.157$. The corresponding band diagrams are calculated along $\Gamma-\mathrm{X}-\mathrm{M}-\Gamma$ symmetry points of the Brillouin Zone (BZ), see Fig. S2 in the supplementary material. Figure 1(b) depicts the theoretical calculation results of EFCs at the fourth band for TM polarization. The superprism section of EFCs is zoomed in the same figure and the directions of Poynting vectors, $\mathbf{S}$, are indicated with arrows. It is well known that for a perfect superprism, sharp edges in EFCs should occur to provide strong beam deflection in terms of small incident angle variations. A strong superprism effect is obtained in the designed $\mathrm{PhCs}$ at the operating frequencies $a$ / $\lambda=0.610-0.635$ and nearly flat EFCs exist that supports a tilted self-collimation effect in that frequency interval, as well. The corresponding tilted self-collimation property exists beyond the symmetry points of BZ so that unwanted 

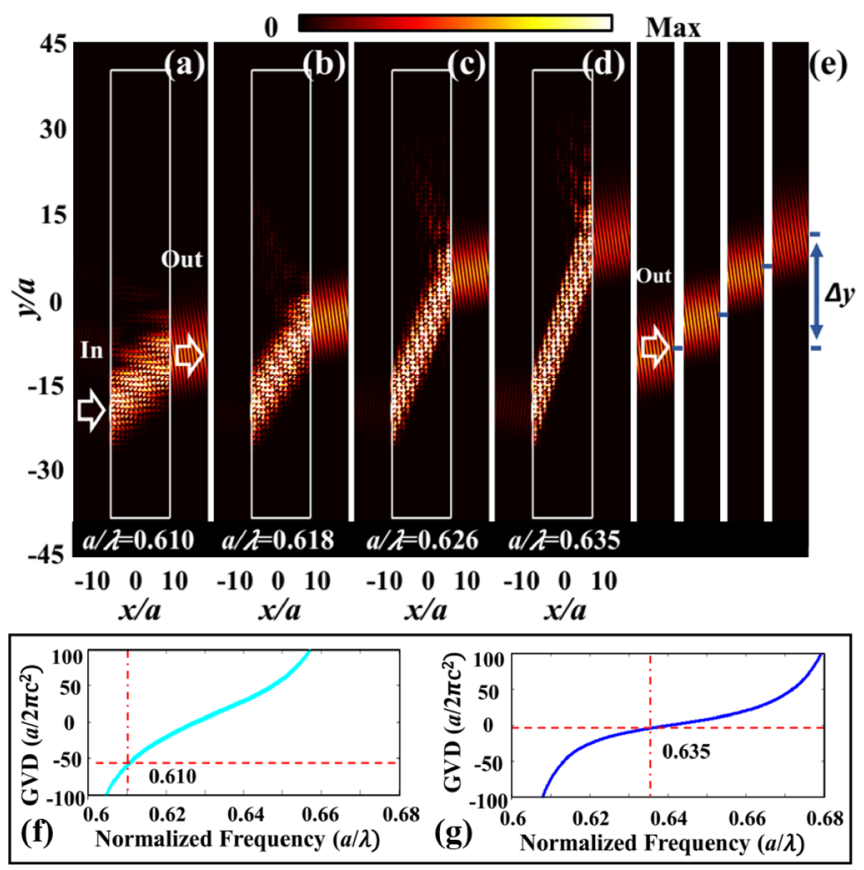

FIG. 2. Electric field intensity distributions of the proposed PhCs calculated at the incident frequencies of (a) $a / \lambda=0.610$, (b) $a / \lambda=0.618$, (c) $a / \lambda$ $=0.626$ and (d) $a / \lambda=0.635$. (e) The cross-sections of output field intensities shown in (a)-(d) are superimposed to better understand generated output beam deflection. (f) and (g) Corresponding GVD calculations at the boundaries of superprism frequencies (see the supplementary material).

beam broadening due to the superprism effect could be compensated. This effect can be observed in group velocity dispersion, $\mathrm{GVD}=\left(\partial^{2} k / \partial \omega^{2}\right)$, which is relatively small and varies from -59 to $0\left(a / 2 \pi c^{2}\right)$ while increasing the operating frequency, to generate a strongly collimated beam for the frequencies used in simulation and experiment. Moreover, calculated dispersion and GVD diagrams are given in Figs. S4 and S5 in the supplementary material.

Spatial electric field intensities for the designed structure are calculated at superprism frequencies of $a / \lambda$ $=0.610-0.635$ with the incidence angle $\theta_{i n}=9^{\circ}$ and the corresponding intensity distributions at the selected operating frequencies of $a / \lambda=\{0.610,0.618,0.626,0.635\}$ are shown in Figs. 2(a)-2(d), respectively. The output cross-sections of the calculated field intensities are superimposed in Fig. 2(e) to better visualize the wavelength-dependent beam shifts in the lateral direction occurring due to $\boldsymbol{S}$-vector superprism nature of the proposed PhCs. The vertical cross-sections of the output field intensities are further calculated and provided as a map plot in Fig. S6(a) of the supplementary material. As can be inferred from Fig. 2(e), a vertical beam shift of $\Delta y=21 a$ for the width of $7 a$ is obtained in the case of $4 \%$ wavelength variation. Relatively smaller GVD values are calculated at superprism frequencies, see Figs. 2(f) and 2(g). It can be inferred from the above results that the propagating beam inside the $\mathrm{PhCs}$ are not exposed to significant beam broadening and different incident frequencies could be spatially separated at the output due to the intrinsic tilted selfcollimation feature of the proposed PhCs.

Propagation angles $\theta_{\text {out }}$ of the low-symmetric $\mathrm{PhCs}$ are analyzed in terms of the incident angle and frequencies to investigate the wavelength as well as the angle sensitivity of the superprism structure, see Fig. 3(a). Calculated $\theta_{\text {out }}$ values range in $\pm 68.9^{\circ}$ in the case of $\theta_{\text {in }}=\left[-10^{\circ}, 10^{\circ}\right]$ within $a / \lambda$ $=0.610-0.635$. As can be understood from Fig. 3(a), the proposed low-symmetric superprism exhibits different angular resolution $\partial \theta_{\text {out }} / \partial \theta_{\text {in }}$ properties: The $\mathrm{PhC}$ structure has very

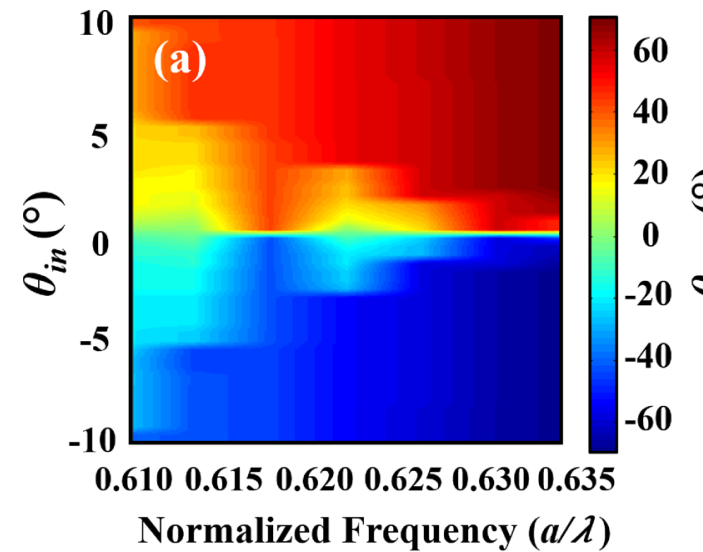

Normalized Frequency $(a / \lambda)$

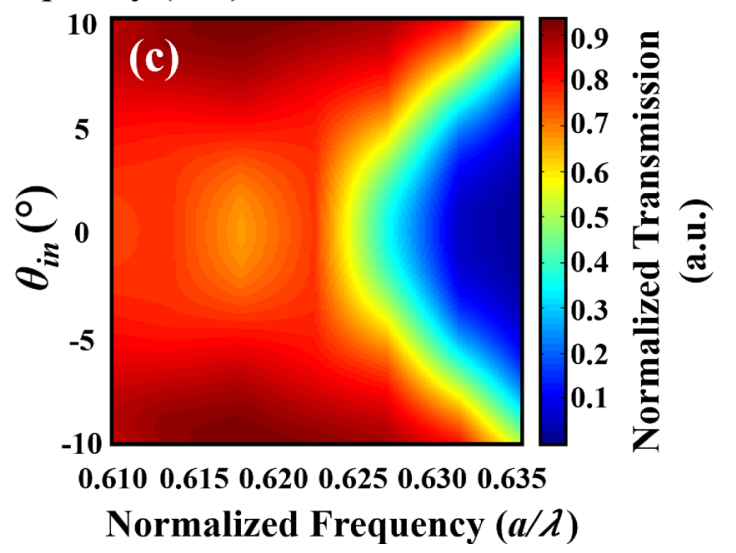

FIG. 3. (a) Propagation angle map with respect to incident angle and operating frequency variations. (b) Propagation angle $\theta_{\text {out }}$ plot at fixed incident frequencies depending on the incident angle variations. (c) Transmission map of the designed PhC superprism structure in terms of the incident angle variation in the range of $\theta_{\text {in }}=\left[-10^{\circ}, 10^{\circ}\right]$. 
high sensitivities in the incident frequencies of $a / \lambda$ $=0.615-0.620$ and $a / \lambda=0.625-0.635$, whereas the angular sensitivity is very low within other frequencies. The corresponding $\theta_{\text {out }}$ values are extracted from the map for fixed frequencies and superimposed in Fig. 3(b).

Four different frequencies are properly selected as $a / \lambda$ $=\{0.610,0.618,0.626,0.635\}$ to better visualize the frequency dependence of the angular sensitivity. As can be seen from Fig. 3(b), the proposed PhCs are very sensitive for small incident angle variations; the corresponding angular resolution equals $\partial \theta_{\text {out }} / \partial \theta_{\text {in }}=\{15.2,1807.4,32.0,1824.8\}$ for the frequencies $a / \lambda=\{0.610,0.618,0.626,0.635\}$. Varying the incident angle to higher values $\theta_{\text {in }}>3.4^{\circ}$, however, the corresponding propagation angle $\theta_{\text {out }}$ remains constant. The underlying reason could be explained from the existing EFCs shown in Fig. 1(b). Above the certain incident angles, the propagating beam is always collimated by the $\mathrm{PhC}$ medium due to its intrinsic collimation property. It can also be inferred from the $\theta_{\text {out }}$ plot in Fig. 3(b) that the angular resolution of the proposed low-symmetric $\mathrm{PhCs}$ is strongly dependent on the incident frequency. Low-loss is another criterion for efficient photonic devices in complex photonic integrated circuits: transmission efficiency at the output channel determines how much power consumption, while light propagates through the $\mathrm{PhC}$ medium. The transmission spectra of the designed superprism structure are calculated with respect to the incident angle variations and superimposed as a transmission map, see Fig. 3(c). The calculated transmission map reveals several crucial remarks about the studied $\mathrm{PhC}$ superprism: In the range of $a / \lambda$ $=0.610-0.625$, the corresponding transmission efficiency is above $59 \%$ for varying incident angles of $\theta_{\text {in }}=\left[-10^{\circ}, 10^{\circ}\right]$ and the maximum calculated transmission is $94 \%$ in this frequency range. In the $a / \lambda=0.625-a / \lambda=0.635$ frequency interval, the transmission efficiencies are calculated to be relatively small. Nevertheless, the transmission efficiency is still above $29 \%$ for $\theta_{\text {in }}>8^{\circ}$, case and at most $90 \%$ transmission is obtained in this frequency range. Moreover, in the case of $\theta_{i n}=9^{\circ}$, the transmission efficiency ranges between $48 \%$ and $93 \%$ depending on the operating frequencies of $a /$ $\lambda=\{0.610-0.635\}$, see Fig. 3(c). In the $a / \lambda=0.610-0.631$ frequency interval, corresponding to $85 \%$ of the operating frequency range, the transmission reaches a value above $80 \%$, see the transmission spectrum given in Fig. S6(c), (see the supplementary material). The obtained calculations indicate that the designed superprism structure could be implemented for efficient photonic wavelength-selective device applications. It is important to note that all Finite-difference time-domain (FDTD) simulations in the study are performed using commercially available LUMERICAL software ${ }^{16}$ with grid sizes of $\mathrm{d} x=\mathrm{d} y=a / 40$.

A microwave experiment is also performed for a reliable demonstration of designed superprism performance. The experimental setup includes an Agilent E5071C type network analyzer, a standard pyramidal horn antenna operating in the frequencies of $8.2 \mathrm{GHz}-12.4 \mathrm{GHz}$, and a monopole antenna as the receiver. The $\mathrm{PhC}$ structure is placed in the near-field of horn antenna to be able to strongly excite the superprism effect inside the PhCs. The horn antenna performs as an incident source with $\theta_{i n}=9^{\circ}$ and the monopole antenna operates as a detector. Cylindrical alumina rods with $\varepsilon_{r}=9.61$ dielectric constant and $3.17 \mathrm{~mm}$ and $6.35 \mathrm{~mm}$ diameters are used in the experiment. The $\mathrm{PhC}$ structure is arranged to be longer than the actual structure designed in the numerical calculations along the vertical direction to observe the intended superprism effect for a wider angle of incidences, see Fig. 4(a). The exact size of the $\mathrm{PhC}$ structure has $34.8 \mathrm{~cm}$ length and $11.1 \mathrm{~cm}$ width and the corresponding lattice constant is calculated as $a=15.85 \mathrm{~mm}$. The microwave setup is surrounded by microwave absorbers to prevent undesired reflections around the system. According to the previous FDTD calculations, the superprism effect was obtained in $a / \lambda=0.610-0.635$ normalized frequencies, which corresponds to the microwave frequency range of $11.55 \mathrm{GHz}-12.02 \mathrm{GHz}$. As can be seen from the output angle plots in Fig. 4(b), the output beam deflection increases with respect to the increase in incident frequencies in the cases of both the microwave experiment and the FDTD calculations. Furthermore, the beam deflection angle at $12.02 \mathrm{GHz}$ is measured to be $\theta_{\text {out }}=58.05^{\circ}$ and as for the numerically calculated value, the output beam deflection was $\theta_{\text {out }}=68.9^{\circ}$. Even though these two values are not close to each other, there is a very similar trend between the experiment and numerical analysis as one can see in Fig. 4(b). Also incident angle variation values are examined in Fig. 4(c). For each normalized frequency $a / \lambda=\{0.610,0.618,0.626,0.635\}$, the maximum output angle values are obtained for $\theta_{\text {out }}$ $=\left\{30.74^{\circ}, 36.01^{\circ}, 48.76^{\circ}, 58.05^{\circ}\right\}$, respectively. According to the output angle values, the angular resolution is calculated as $\partial \theta_{\text {out }} / \partial \theta_{\text {in }}=\{23.39,28.1,41.17,55.58\}$, for normalized frequencies $a / \lambda=\{0.610,0.618,0.626,0.635\}$, respectively, for $1^{\circ}$ change of incident angle. Besides all these investigations, experimental transmission mapping of the structure is performed in Fig. 4(d). According to the results obtained, the maximum transmission was reached $72.4 \%$ at $\theta_{\text {in }}=9^{\circ}$ input angle and at $11.7 \mathrm{GHz}$. The lowest transmission value was obtained at a frequency of $11.96 \mathrm{GHz}$ at $9^{\circ}$ input angle with a value of $21.5 \%$.

Contrary to other studies, ${ }^{4,10,17-19}$ the presented study proposes a self-collimated superprism effect without any structural modifications. The designed superprism structure also provides high wavelength sensitivity performance, i.e., large deflected angle variations for different frequencies with relatively high transmission as shown in Fig. 4(b), which is critically important for Wavelength Demultiplexing (WDM) applications in optical communications. ${ }^{19}$

All the discussed performance analyses are presented in Table I. Taking all these results into consideration, although the best results are not being obtained for some parameters, the overall results in the present work are better. Accordingly, the designed structure provides a superprism effect with a high performance according to the numerical and experimental results presented in this study.

In conclusion, a high wavelength sensitive superprism structure is designed which possesses tilted self-collimation behavior, which is proven by calculated GVD values that decrease exponentially from -59 to $0\left(a / 2 \pi c^{2}\right)$, in $a / \lambda$ $=0.610-0.635$ interval. A theoretical approach to the bandstructure of low-symmetric PhCs is performed to achieve such a considerable beam deflection effect with wide-angle 

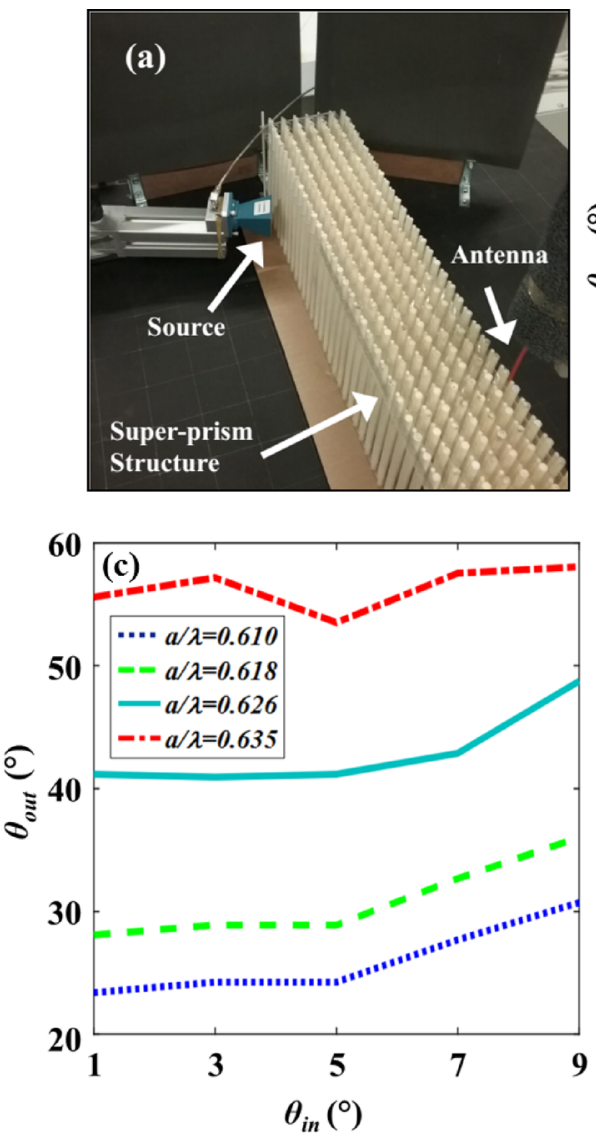

FIG. 4. (a) Superprism experimental setup with a microwave source, antenna, and alumina rods. The setup is surrounded by microwave absorbers to eliminate environmental noises. (b) Beam deflection angle plot in the cases of the microwave experiment and the FDTD calculation. (c) Propagation angle $\theta_{\text {out }}$ plot at incident frequencies depending on the incident angle variations for experimental outcomes. (d) Transmission map of the designed $\mathrm{PhC}$ structure according to experimental measurements $\theta_{\text {in }}=1^{\circ}, 3^{\circ}, 5^{\circ}, 7^{\circ}, 9^{\circ}$ incident angles.
TABLE I. The comparison of various studies according to the superprism figure of merits.

\begin{tabular}{lccc}
\hline \hline Study & $\begin{array}{c}\text { Wavelength } \\
\text { sensitivity }\end{array}$ & Compactness & Transmission \\
\hline Proposed work & $4.0 \%-40^{\circ} /$ & $7 \times 28 \mu \mathrm{m}^{2} /$ & $\sim 90.0 / 72.4$ \\
(Sim./Exp.) & $4.0 \%-27.3^{\circ}$ & $11.1 \times 34.8 \mathrm{~cm}^{2}$ & \\
Matsumoto $^{4}$ (Sim.) & $4.7 \%-27^{\circ}$ & $0.2 \mathrm{~mm}^{2}$ & 79.4 \\
Pahlavan $^{10}$ (Sim.) & $1.9 \%-133^{\circ}$ & $1.15 \times 5.76 \mu \mathrm{m}^{2}$ & 64 \\
Căbuz $^{17}$ (Sim.) & Single freq. & $\ldots$ & $\sim 80.0$ \\
Matsumoto $^{18}$ (Exp.) & $3.9 \%-15^{\circ}$ & $80 \times 100 \mu \mathrm{m}^{2}$ & $\ldots$ \\
$\mathrm{Li}^{19}$ (Sim.) & $2.7 \%-54^{\circ}$ & $10 \mu \mathrm{m}^{2}$ & 55.0 \\
\hline \hline
\end{tabular}

magnification from $29.1^{\circ}$ to $68.9^{\circ}$ deflection angle. The proposed low symmetric superprism $\mathrm{PhCs}$ have a high wavelength selectivity, which enables designing highly efficient wavelength demultiplexers. The transmission efficiency of the $\mathrm{PhC}$ structure varying between $48 \%$ and $93 \%$ at the incident angle of $\theta_{i n}=9^{\circ}$ within the superprism frequencies indicates that the proposed structure is feasible for highly efficient photonic device implementations. Experimental verification of the intended $\mathbf{S}$-vector superprism effect is also carried out in the microwave frequencies, in which case the experimental measurements match quite well with the corresponding FDTD calculations.

See supplementary material for analytical derivations of EFCs and systematical comparison of the $\mathrm{C} 1$ symmetry configuration with other rotational symmetries where the symmetry reduction effect on the EFCs is analyzed and also for the additional performance analysis of the self-collimated superprism effect for its ideal incident angle.
The authors M.G. and H.K. gratefully acknowledge the financial support of the Scientific and Technological Research Council of Turkey (TUBITAK) with Project No. 115R036. H.K. also acknowledges the partial support of the Turkish Academy of Sciences.

${ }^{1}$ S. N. Tandon, M. Soljačić, G. S. Petrich, J. D. Joannopoulos, and L. A. Kolodziejski, Photonics Nanostruct. 3, 10 (2005).

${ }^{2}$ H. Kosaka and T. Kawashima, Phys. Rev. B 58, R10096 (1998).

${ }^{3}$ T. Matsumoto and T. Baba, IEICE Trans. Electron. E87-C, 393 (2004).

${ }^{4}$ T. Matsumoto, S. Fujita, and T. Baba, Opt. Express 13, 10768 (2005).

${ }^{5}$ C. Luo, M. Soljacić, and J. D. Joannopoulos, Opt. Lett. 29, 745 (2004).

${ }^{6}$ J. Dellinger, D. Bernier, B. Cluzel, X. Le Roux, A. Lupu, F. de Fornel, and E. Cassan, Opt. Lett. 36, 1074 (2011).

${ }^{7}$ T. Baba and T. Matsumoto, Appl. Phys. Lett. 81, 2325 (2002).

${ }^{8}$ M. J. Steel, R. Zoli, C. Grillet, R. C. McPhedran, C. Martijn De Sterke, A. Norton, P. Bassi, and B. J. Eggleton, Phys. Rev. E 71, 056608 (2005).

${ }^{9}$ B. Gao, Z. Shi, and R. W. Boyd, Opt. Express 23, 6491 (2015).

${ }^{10}$ S. Pahlavan and V. Ahmadi, IEEE Photonics Technol. Lett. 29, 511 (2017).

${ }^{11}$ M. Turduev, I. H. Giden, and H. Kurt, Photonics Nanostruct. 11, 241 (2013).

${ }^{12}$ M. Plihal, A. Shambrook, A. A. Maradudin, and P. Sheng, Opt. Commun. 80, 199 (1991).

${ }^{13}$ X. H. Wang, B. Y. Gu, Z. Y. Li, and G. Z. Yang, Phys. Rev. B 60, 11417 (1999).

${ }^{14}$ K. M. Leung and Y. F. Liu, Phys. Rev. Lett. 65, 2646 (1990).

${ }^{15}$ MATLAB and Statistics Toolbox Release (Mathworks, Inc., Natic, MA, 2015).

${ }^{16}$ Lumerical FDTD Solutions, Inc., http://www.lumerical.com for high performance FDTD-method Maxwell solver for the design, analysis and optimization of nanophotonic devices, processes and materials.

${ }^{17}$ A. I. Căbuz, E. Centeno, and D. Cassagne, Appl. Phys. Lett. 84, 2031 (2004).

${ }^{18}$ T. Matsumoto, T. Asatsuma, and T. Baba, Appl. Phys. Lett. 91, 091117 (2007).

${ }^{19}$ W. Li, X. G. Zhang, X. L. Lin, and X. Y. Jiang, Opt. Lett. 39, 4486 (2014). 